DrAFt VERSION July 6, 2021

Preprint typeset using $\mathrm{LAT}_{\mathrm{E}} \mathrm{X}$ style emulateapj v. 12/16/11

\title{
CONSTRAINING THE ENVIRONMENTAL PROPERTIES OF FRB 131104 USING THE UNIFIED DYNAMICAL AFTERGLOW MODEL
}

\author{
Zong-Kai Peng ${ }^{1,2}$, Shan-Qin Wang ${ }^{1,2,3}$, Liang-Duan Liu ${ }^{1,2,4}$, Zi-Gao Dai ${ }^{1,2}$, And Hai Yu ${ }^{1,2,5}$ \\ ${ }^{1}$ School of Astronomy and Space Science, Nanjing University, Nanjing 210093, China; dzg@nju.edu.cn \\ ${ }^{2}$ Key Laboratory of Modern Astronomy and Astrophysics (Nanjing University), Ministry of Education, China \\ ${ }^{3}$ Department of Astronomy, University of California, Berkeley, CA 94720-3411, USA \\ ${ }^{4}$ Department of Physics and Astronomy, University of Nevada, Las Vegas, NV 89154, USA and \\ ${ }^{5}$ Department of Physics, Kansas State University, 116 Cardwell Hall, Manhattan, KS 66506, USA \\ Draft version July 6, 2021
}

\begin{abstract}
Multi-band observations of the fast radio burst (FRB) 131104 show that this burst may be associated with a gamma-ray transient entitled Swift J0644.5-5111. Follow-up observations for potential X-ray and radio counterparts of FRB 131104/Swift J0644.5-5111 got null results and provided the upper limits of the emission flux at $5.5 \mathrm{GHz}, 7.5 \mathrm{GHz}, U$-band, and X-ray band. By assuming this association and using these upper limits, environmental properties (the fraction of energy in a magnetic field $\varepsilon_{\mathrm{B}}$ and the number density $n$ ) of the progenitor system of FRB 131104/Swift J0644.5-5111 were constrained in the context of the standard afterglow model that neglects the non-relativistic effect and jet effect by several groups. In this paper, we adopt a unified afterglow model that takes into account the non-relativistic effect and jet effect and use the upper limits of four bands $(5.5 \mathrm{GHz}, 7.5$ $\mathrm{GHz}, U$-band, and X-ray) to obtain more stringent constraints on the parameter space spanned by $\varepsilon_{\mathrm{B}}$ and $n$. We thus suggest that FRB 131104/Swift J0644.5-5111 might originate from a black holeneutron star merger event. Moreover, we calculate multi-band emissions from a kilonova powered by the radioactivity of $r$-process elements synthesized in the ejected neutron-rich material and find that the $U$-band emission from the putative kilonova is significantly lower than the upper limit of the observations.

Subject headings: gamma-ray burst: general - radio continuum: general
\end{abstract}

\section{INTRODUCTION}

Fast radio bursts (FRBs) discovered one decade ago are radio transients whose duration timescale is only a few milliseconds (Lorimer et al. 2007; Ravi et al. 2016; Kulkarni et al. 2014). It seems that the event rate of FRBs is very low since only 34 FRBs have been discovered. ${ }^{1}$ Considering the facts that radio telescopes observe a small area of sky ${ }^{2}$ and that the observed band is rather narrow, however, the actual explosion rate of FRBs must be rather high, about $2.8 \times 10^{3} \mathrm{Gpc}^{-3} \mathrm{yr}^{-1}$, and about $10^{4}$ bursts per day all sky (Hassall et al. 2013). These FRBs may be divided into two subclasses, repeating and non-repeating FRBs. Except for FRB 121102 which is a repeating FRB that has been recorded more than 140 bursts (cf. Palaniswamy et al. 2018), ${ }^{3}$ and the other are non-repeating. ${ }^{4}$

The typical frequency and the observed peak flux of FRBs are $\sim 1 \mathrm{GHz}$ and $\sim 1 \mathrm{Jy}$, respectively. ${ }^{5}$ Based on

\footnotetext{
${ }^{1}$ http://www.astronomy.swin.edu.au/pulsar/frbcat/

2 The total instantaneous field-of-view (FoV) of the Molonglo Observatory Synthesis Telescope (UTMOST) is $\sim 8 \mathrm{deg}^{2}$ (http://astronomy.swin.edu.au/research/utmost/?page_id=32); the total instantaneous FoV of the Five-hundred-meter Aperture Spherical radio Telescope (FAST) is $0.15 \operatorname{deg}^{2}$ (Li et al. 2017), and based on the method provided by $\mathrm{Li}$ et al. (2017), the computed total instantaneous FoV of the Parkes radio telescope is $2.24 \mathrm{deg}^{2}$.

3 Most of the bursts have not been published to date, see, Spitler, the 2017 Aspen FRB conference (http://aspen17.phys.wvu.edu/Spitler.pdf).

4 Piro \& Burke-Spolaor (2017) proposed that FRB 110220 and FRB 140514 might be the two bursts originated a repeating FRB since their positions are close to each other.

${ }^{5}$ However, the peak fluxes of some FRBs are significantly higher
}

these facts, the inferred brightness temperature can be up to $\sim 10^{37} \mathrm{~K}$, which is most likely to be reproduced by the coherent radiation mechanism (Lorimer et al. 2007; Katz 2014; Yang \& Zhang 2017). The dispersion measure (DM) of a FRB can be calculated from the difference of the arrival time between different frequencies. By comparing the DMs of the FRBs and that of our Galaxy at the same directions, one can find that the former are significantly larger than the latter (Thornton et al. 2013). Research for the distributions of observed FRBs indicate that the positions of these FRBs are random rather than concentrating to the disk of the Galaxy (Caleb et al. 2017), indicating that the FRBs have a cosmological origin rather than a Galactic origin.

In the past decade, many theoretical models have been proposed to explain FRBs, including magnetar giant flares (Kulkarni et al. 2014), giant pulses from young pulsars (Connor et al. 2016), planetary companion around pulsars (Mottez \& Zarka 2014), neutron star (NS)-black hole (BH) transitions (Falcke \& Rezzolla 2014), NSwhite dwarf (WD) mergers (Gu et al. 2016), NS-NS mergers (Abadie et al. 2010; Wang et al. 2016), WDWD mergers (Kashiyama et al. 2013), NS-BH mergers (Mingarelli et al. 2015), pulsar-asteroid collisions (Geng \& Huang 2015; Dai et al. 2016b), cosmic string collisions (Zadorozhna 2015), charged BH-BH mergers (Zhang 2016), light sails of extragalactic civilizations (Lingam \& Loeb 2017), and so on.

Searching and observing the electromagnetic counter-

than 1 Jy, e.g., the peak flux of FRB 150807 is $128 \pm 5 \mathrm{Jy}$ (Ravi et al. 2016). 
parts at other bands, e.g., X-ray, ultraviolet (UV), optical, as well as infrared (IR), are helpful for determining the nature of FRBs. However, discovering these electromagnetic counterparts of FRBs are very difficult since the positional accuracy for FRBs are very poor so that their host galaxies can hardly be fixed. Furthermore, a major of FRBs were found from historical records and the electromagnetic counterparts at other bands cannot be confirmed.

Nevertheless, two (FRB 150418 and FRB 131104) of 33 non-repeated FRBs might have electromagnetic counterpart candidates at other bands observed. Keane et al. (2016) declared that they found bright radio transients on $5.5 \mathrm{GHz}$ and $7.5 \mathrm{GHz}$ from 2 hours to 6 days after with FRB 150418. The results of FRB 150418 are controversial since the observations of the Very Large Array (VLA) suggested that the $5.5 \mathrm{GHz}$ and $7.5 \mathrm{GHz}$ radio emission detected by the Australia Telescope Compact Array (ATCA) 2 hours to 6 days after the trigger of FRB 150418 might be generated by the activity of an active galactic nucleus (AGN) rather than FRB 150418 (Williams \& Berger 2016).

A gamma-ray transient, Swift J0644.5-5111, has been reported to be associated with FRB 131104, the confidence level is $3.2 \sigma$ (DeLaunay et al. 2016). The duration $T_{90}$ and the fluence $S_{\gamma}$ of Swift J0644.5-5111 are $\approx 377 \mathrm{~s}$ and $\approx 4 \times 10^{-6} \mathrm{erg} \mathrm{cm}^{-2}$, respectively; the DM of FRB 131104 is $779 \pm 1 \mathrm{pc} \mathrm{cm}^{-3}$. The redshift $(z)$ of FRB 131104/Swift J0644.5-5111 inferred from the $\mathrm{DM}$ is $\approx 0.55 .^{6}$ Therefore, the isotropic gamma-ray energy $E_{\gamma \text {,iso }}$ of Swift J0644.5-5111 is $\approx$ $5 \times 10^{51}$ erg (DeLaunay et al. 2016). The follow-up observations performed by the XRT and the UVOT on board Swift two days after Swift was triggered by Swift J0644.5-5111 didn't detect X-ray and optical counterparts (DeLaunay et al. 2016). Shannon \& Ravi (2017) observed the $5.5 \mathrm{GHz}$ and $7.5 \mathrm{GHz}$ counterparts of FRB 131104 three days to 2.5 years after the FRB trigger and found that the upper limits of the $5.5 \mathrm{GHz}$ and $7.5 \mathrm{GHz}$ are $70 \mu \mathrm{Jy}$ and $100 \mu \mathrm{Jy}$, respectively.

A powerful tool to determine the progenitor system of Swift J0644.5-5111 is to research the properties of its environment, e.g., the number density $(n)$ of the interstellar medium (ISM) surrounding the progenitor. Assuming that Swift J0644.5-5111 was associated with FRB 131104 and using the upper limit of $5.5 \mathrm{GHz}$ counterpart of FRB 131104 reported by Shannon \& Ravi (2017), Murase et al. (2017) constrained the density of the ISM of FRB 131104 with the external shock model of afterglows and concluded that $n \lesssim 2 \times 10^{-3} \mathrm{~cm}^{-3}$. By using the same model and the upper limits of radio counterparts and adopting different values of redshift, the fraction of energy in magnetic field $\left(\varepsilon_{\mathrm{B}}\right)$, and the fraction of energy in electrons $\left(\varepsilon_{\mathrm{e}}\right)$, Gao \& Zhang (2017) constrained the parameter space of the ISM density. According to the detected limit obtained by the XRT and employing the afterglow model, Dai et al. (2016a) got a stringent constraint, $n \lesssim 2.6 \times 10^{-4} \mathrm{~cm}^{-3}$.

While some research mentioned above (Murase et al. 2017; Gao \& Zhang 2017; Dai et al. 2016a) well con-

\footnotetext{
6 The inferred redshift relies on the assumption that the DM is dominated by the intergalactic medium. A large host contribution would result in a smaller redshift.
}

strained the density of the ISM of Swift J0644.55111/FRB 131104, they assumed that the explosion producing the gamma-ray burst (GRB) is isotropic and relativistic, and didn't take into account the jet effect and the evolution from ultra-relativistic to non-relativistic. However, these two effects cannot be neglected since the outflows launched by the explosions of a massive star or the merger of NS-NS/BH binaries are jets rather than isotropic fireballs, and the ISM would decelerate the relativistic jet and the jet would eventually become nonrelativistic if the jet sweeps up an enough mass of the ISM.

In this paper, we also suppose that both FRB 131104 and Swift J0644.5-5111 originate from an explosion of a massive star or a merging of NS-NS/BH binary and adopt the unified dynamic afterglow model proposed by Huang et al. (1999, 2000a,b) which describes ultrarelativistic, trans-relativistic, and non-relativistic outflows to pose more stringent constraints on the density of the ISM and other parameters of Swift J0644.55111/FRB 131104. The jet effect and non-relativistic effect would be taken into account throughout this paper. The remainder of this paper is organized as follows. In Section 2 we present our adopted model. In Section 3 , we constrain the parameters. Our discussion and conclusions are presented in Sections 4 and 5, respectively.

\section{THE MODEL}

We assume that FRB 131104 is associated with Swift J0644.5-5111 and originates from the core collapse of a massive star or the merger of binary compact stars. It is suggested that a relativistic outflow could be generated during these processes. The relativistic outflow whose velocity is significantly larger than the sound velocity of the ISM sweeps up this external medium, producing a forward shock which accelerates the electrons. The accelerated electrons emit photons which constitute multi-band afterglows (Sari et al. 1998). In order to accurately describe the outflow evolution from ultrarelativistic to non-relativistic stages, Huang et al. (1999, 2000a,b) developed a unified dynamic model for an afterglow which can be applied both the completely adiabatic case and the completely radiative case. The equation of the dynamic evolution is given by

$$
\frac{d \gamma}{d m}=-\frac{\gamma^{2}-1}{M_{\mathrm{ej}}+\epsilon m+2(1-\epsilon) \gamma m},
$$

where $\gamma$ is the Lorentz factor of the shock wave, $m$ is the mass of the ISM swept up by the shock, and $M_{\mathrm{ej}}$ and $\epsilon$ are the outflow mass and the radiative efficiency respectively. To solve this equation, three additional auxiliary equations are required by

$$
\begin{aligned}
\frac{d R}{d t} & =\beta c \gamma\left(\gamma+\sqrt{\gamma^{2}-1}\right), \\
\frac{d m}{d R} & =2 \pi R^{2}(1-\cos \theta) n m_{\mathrm{p}}, \\
\frac{d \theta}{d t} & =\frac{c_{s}\left(\gamma+\sqrt{\gamma^{2}-1}\right)}{R},
\end{aligned}
$$

where $\theta$ is the half-angle of the outflow, $c_{s}$ is the sound velocity of the outflow, $R$ is the radius of the shock, and 
$t$ is the observed time. The dynamic evolution of the shock can be obtained by solving these equations.

According to the standard afterglow model, the distribution of the electrons accelerated by the forward shock is a power-law function of $\gamma_{\mathrm{e}}$ (Mészáros \& Rees 1997; Sari et al. 1998): $N_{\mathrm{e}}\left(\gamma_{\mathrm{e}}\right) \mathrm{d} \gamma_{\mathrm{e}} \propto \gamma_{\mathrm{e}}^{-p} \mathrm{~d} \gamma_{\mathrm{e}}$, where $p$ is the spectral index and is fixed to be 2.5 here. The minimum Lorentz factor of the accelerated electrons is

$$
\gamma_{\mathrm{m}}=\frac{p-2}{p-1} \frac{m_{\mathrm{p}}}{m_{\mathrm{e}}} \varepsilon_{\mathrm{e}}(\gamma-1)+1,
$$

where $m_{\mathrm{p}}$ and $m_{\mathrm{e}}$ are the masses of the protons and electrons, respectively. $\varepsilon_{\mathrm{e}}$ is the fraction of the shock energy going into the electrons. The critical Lorentz factor above which the relativistic electrons would emit synchrotron radiation in the dynamical time $t$ is

$$
\gamma_{\mathrm{c}}=(1+z) \frac{6 \pi m_{\mathrm{e}} c}{\sigma_{\mathrm{T}} \gamma B^{2} t},
$$

where $c$ is the speed of light in a vacuum, $\sigma_{\mathrm{T}}$ is the Thomson scattering cross-section, $B=\left(32 \pi m_{\mathrm{p}} \varepsilon_{\mathrm{B}} n\right)^{\frac{1}{2}} \gamma c$ is the magnetic field behind the shock and $\varepsilon_{\mathrm{B}}$ is the fraction of energy in a magnetic field. Hence, the two characteristic frequencies of the synchrotron radiation are

$$
\nu_{\mathrm{m}}=3.3 \times 10^{11}(1+z)^{\frac{1}{2}} \varepsilon_{\mathrm{e},-1}^{2} \varepsilon_{\mathrm{B},-2}^{\frac{1}{2}} E_{51.7}^{\frac{1}{2}} t_{\text {day }}^{-\frac{3}{2}} \mathrm{~Hz},
$$

and

$$
\nu_{c}=3.8 \times 10^{17}(1+z)^{-\frac{1}{2}} \varepsilon_{B,-2}^{-\frac{3}{2}} E_{51.7}^{-\frac{1}{2}} n_{-2}^{-1} t_{\text {day }}^{-\frac{1}{2}} \mathrm{~Hz} .
$$

Assuming that $\varepsilon_{\mathrm{e}}=0.1$ and $\varepsilon_{\mathrm{B}}=0.01, \nu_{m}$ becomes smaller than $\nu_{c}$ very quickly. The electrons would be in the slow-cooling phase about two days after the explosion. Considering the synchrotron self-absorption effect, the synchrotron self-absorption frequency at the radio band is

$$
\nu_{a}=\left\{\begin{array}{cr}
2.4 \times 10^{8}(1+z)^{-1} \varepsilon_{e,-1}^{-1} \varepsilon_{B,-2}^{\frac{1}{5}} E_{51.7}^{\frac{1}{5}} n_{-2}^{\frac{3}{5}} \mathrm{~Hz} \\
\nu_{a}<\nu_{m}<\nu_{c} \\
8.6 \times 10^{9}(1+z)^{\frac{p-6}{2(p+4)}} \varepsilon_{e,-1}^{\frac{2(p-1)}{p+4}} \varepsilon_{B,-2}^{\frac{p+2}{2(p+4)}} E_{51.7}^{\frac{p+2}{2(p+4)}} \\
\times n_{-2}^{\frac{2}{p+4}} t_{\text {day }}^{-\frac{3 p+2}{2(p+4)}} \mathrm{Hz}, & \nu_{m}<\nu_{a}<\nu_{c}
\end{array}\right.
$$

the observed flux is

$$
F_{\nu}= \begin{cases}\left(\frac{\nu}{\nu_{a}}\right)^{2}\left(\frac{\nu_{a}}{\nu_{m}}\right)^{\frac{1}{3}} F_{\nu, \max }, & \nu<\nu_{a} \\ \left(\frac{\nu}{\nu_{m}}\right)^{\frac{1}{3}} F_{\nu, \max }, & \nu_{a}<\nu<\nu_{m} ; \\ \left(\frac{\nu}{\nu_{m}}\right)^{-\frac{p-1}{2}} F_{\nu, \max }, & \nu_{m}<\nu<\nu_{c} \\ \left(\frac{\nu}{\nu_{c}}\right)^{-\frac{p}{2}}\left(\frac{\nu_{c}}{\nu_{m}}\right)^{-\frac{p-1}{2}} F_{\nu, \max }, & \nu_{c}<\nu<\nu_{\max }\end{cases}
$$

when $\nu_{a}<\nu_{m}<\nu_{c}$, and

$$
F_{\nu}= \begin{cases}\left(\frac{\nu}{\nu_{m}}\right)^{2}\left(\frac{\nu_{m}}{\nu_{a}}\right)^{\frac{p+4}{2}} F_{\nu, \max }, & \nu<\nu_{m} \\ \left(\frac{\nu}{\nu_{a}}\right)^{\frac{5}{2}}\left(\frac{\nu_{a}}{\nu_{m}}\right)^{-\frac{p-1}{2}} F_{\nu, \max }, & \nu_{m}<\nu<\nu_{a} \\ \left(\frac{\nu}{\nu_{m}}\right)^{-\frac{p-1}{2}} F_{\nu, \max }, & \nu_{a}<\nu<\nu_{c} \\ \left(\frac{\nu}{\nu_{c}}\right)^{-\frac{p}{2}}\left(\frac{\nu_{c}}{\nu_{m}}\right)^{-\frac{p-1}{2}} F_{\nu, \max }, & \nu_{c}<\nu<\nu_{\max }\end{cases}
$$

when $\nu_{m}<\nu_{a}<\nu_{c}$. Here, $\nu_{\max }$ is the characteristic frequency of the electron having the largest Lorentz factor $\gamma_{\max } \simeq\left[6 \pi q_{\mathrm{e}} /\left(\sigma_{\mathrm{T}} B\right)\right]^{1 / 2} . F_{\nu, \max }$ is the peak value of the observed flux

$$
\begin{aligned}
F_{\nu, \max } & =(1+z) \frac{N_{e, \text { tot }} P_{\nu, \max }}{4 \pi D_{L}^{2}} \\
& =5.7 \times 10^{2}(1+z) \varepsilon_{B,-2}^{\frac{1}{2}} E_{51.7} n_{-2}^{\frac{1}{2}} D_{L, 28}^{-2} \mu \mathrm{Jy},
\end{aligned}
$$

where $D_{L}$ is the luminosity distance to the source.

Some relevant works (e.g., Murase et al. 2017; Gao \& Zhang 2017; Dai et al. 2016a) in the literature do not take into account the non-relativistic effect, the jet effect and the lateral expansion effect. However, all these effects are included in our numerical calculations.

\section{CONSTRAINING THE PARAMETERS}

In this section, we use the equations of the unified dynamic afterglow model listed in section 2 as well as the upper limits of the observations of radio $(5.5 \mathrm{GHz}$ and $7.5 \mathrm{GHz}), U$-band, and X-ray counterparts to constrain the ISM density $(n)$ and $\varepsilon_{\mathrm{B}}$. Some parameters are fixed: $z=0.55, E_{\gamma, \text { iso }}=5 \times 10^{51} \mathrm{erg}, \theta=0.1, p=2.5, \varepsilon_{\mathrm{e}}=0.1$. Thus, the light curves depend on the values of $\varepsilon_{\mathrm{B}}$ and $n$ which are the parameters needed to be constrained. For comparison, we also calculate the multi-band afterglows from both for the isotropic outflow and for the jet whose half-angle is supposed to be 0.1 here. Santana et al. (2014) presented a systematic study on magnetic fields in GRB external forward shocks and found that the range and the median value of $\varepsilon_{\mathrm{B}}$ are $10^{-4}-10^{-0.5}$ and $\approx 10^{-2}$, respectively. Hence, we set $\varepsilon_{\mathrm{B}}=0.0001,0.001,0.01$, and 0.1 .

\subsection{The parameters inferred from the models}

The parameter limits derived from the unified dynamic model are listed in Table 1 and the theoretical light curves are plotted in Fig. 1-4. We emphasize that the $U$-band emission is fainter than observations even if $\varepsilon_{\mathrm{B}}=0.1$ and $n=1 \mathrm{~cm}^{-3}$, indicating that the upper limit of $U$-band observations cannot be used to constrain the relevant parameters.

It should be noted that some papers (e.g., Dai et al. 2016a, Murase et al. 2017, and Gao \& Zhang 2017) have constrained the ISM density for the isotropic outflow case. For example, Dai et al. (2016a) used the upper limit of the X-ray observations and inferred that $n \lesssim 2.6 \times 10^{-4} \mathrm{~cm}^{-3}$ if $\varepsilon_{B}=0.01$, being roughly consistent with the value inferred here $\left(2.0 \times 10^{-4} \mathrm{~cm}^{-3}\right)$.

According to Table 1, the ISM density derived by the model that takes into account the jet effect and the nonrelativistic effect is apparently different from that derived by the isotropic models adopted by previous studies (e.g., Dai et al. 2016a, Murase et al. 2017, and Gao \& Zhang 2017). Moreover, the constraint on the ISM density obtained by multi-band upper limits is tighter than that derived by any single band. These two facts demonstrate the necessity of employing the unified model and combining the data at all bands.

We give Table 2 to describe the properties adopted by four models (this paper, Dai et al. 2016a, Murase et al. 2017, and Gao \& Zhang 2017) and their results.

\subsection{The contours}


TABLE 1

Parameter limits Derived From the Unified dynamic Model

\begin{tabular}{ccccc}
\hline \hline$\varepsilon_{\mathrm{B}}$ & $\begin{array}{c}n\left(\mathrm{~cm}^{-3}\right) \\
5.5 \mathrm{GHz}\end{array}$ & $\begin{array}{c}n\left(\mathrm{~cm}^{-3}\right) \\
7.5 \mathrm{GHz}\end{array}$ & $\begin{array}{c}n\left(\mathrm{~cm}^{-3}\right) \\
\text { X-ray }\end{array}$ & $\begin{array}{c}n\left(\mathrm{~cm}^{-3}\right) \\
\text { Together }\end{array}$ \\
\hline \hline Jet & & & & \\
\hline 0.0001 & non & non & non & non \\
0.001 & $<6.5 \times 10^{-2}$ & $<1.6 \times 10^{-1}$ & non & $<6.5 \times 10^{-2}$ \\
0.01 & $<6.6 \times 10^{-3}$ & $<1.3 \times 10^{-2}$ & $<7.4 \times 10^{-4}$ & $<7.4 \times 10^{-4}$ \\
& & or $>1.9$ & \\
0.1 & $<7.4 \times 10^{-4}$ & $<1.3 \times 10^{-3}$ & $<6.3 \times 10^{-5}$ & $<6.3 \times 10^{-5}$ \\
& & & or $>5.3$ & \\
\hline \hline Isotropic & & & & \\
\hline 0.0001 & $<6.5 \times 10^{-2}$ & $<1.3 \times 10^{-1}$ & $<1.0$ & $<6.5 \times 10^{-2}$ \\
0.001 & $<9.0 \times 10^{-3}$ & $<1.0 \times 10^{-2}$ & $<1.0 \times 10^{-2}$ & $<9.0 \times 10^{-3}$ \\
0.01 & $<1.0 \times 10^{-3}$ & $<1.5 \times 10^{-3}$ & $<2.0 \times 10^{-4}$ & $<2.0 \times 10^{-4}$ \\
0.1 & $<8.5 \times 10^{-5}$ & $<1.0 \times 10^{-4}$ & $<3.0 \times 10^{-6}$ & $<3.0 \times 10^{-6}$ \\
\hline \hline
\end{tabular}

TABLE 2

The DifFerences of OUR MODEL AND THREE PREVIOUS MODELS

\begin{tabular}{ccccc}
\hline \hline & this paper & Murase17 & Gao17 & Dai16 \\
\hline \hline Isotropic & yes & yes & yes & yes \\
Jet & yes & no & no & no \\
Relativistic & yes & yes & yes & yes \\
Non-relativistic & yes & no & no & no \\
kilonova & yes & no & no & no \\
X-ray & yes & no & yes & yes \\
Optical & yes & no & no & no \\
Radio & yes & yes & yes & yes \\
\hline \hline
\end{tabular}

Fig. 5 consists of four contour sub-figures corresponding to the detected flux at $5.5 \mathrm{GHz}(t=3$ days), $7.5 \mathrm{GHz}$ $(t=3$ days), $U$-band ( $t=2$ days), and X-ray $(t=2$ days). The red lines represent the detection limits. Every panel has $48 \times 48$ data points each of which represents the value of $\varepsilon_{\mathrm{B}}$ and $n$ at this point. The curves in these panels represent the detection limits, and the admitted (two dimensional) parameter space is lower than the lines. Panel c (corresponding to $U$-band) has no line, indicating that the theoretical $U$-band flux reproduced by all possible parameters are smaller than the detected limit and cannot pose any constraint on the parameters.

\section{DISCUSSION}

4.1. The possible origin of Swift J0644.5-5111/FRB 131104

It has long been believed that the GRB prompt emission would be produced in the relativistic jet launched by the core collapse of massive stars (Usov 1992; Woosley 1993; Thompson \& Duncan 1995; MacFadyen \& Woosley 1999) or mergers of compact star systems containing at least one neutron star. The GRBs are usually divided into two categories: long GRBs with $T_{90}>2 \mathrm{~s}$ are linked to the core collapse of massive stars while short GRBs with $T_{90}<2$ s are linked to the mergers of binary compact stars. However, some long GRBs (e.g., GRB 060614, Yang et al. 2015) share the properties of short GRBs and can be classified to "longshort bursts" which might be produced by NS-NS mergers or BH-NS mergers. The massive star scenario can be excluded both because the required medium density is very low (as in section 3) and because the association of
Swift J0644.5-5111/FRB 131104 requires that the FRB was emitted before the gamma-ray radiation but the scenario gives the reverse time sequence (Gao \& Zhang 2017). Therefore, the possibility that Swift J0644.55111 is a "long-short burst" produced by an NS-NS/BH merger event must be investigated. Murase et al. (2017) have suggested that Swift J0644.5-5111 might be associated with a gamma-ray burst and discussed various possible origins of Swift J0644.5-5111 (including the possibility that it might be associated with an NSNS/BH merger). Here, we discuss the possible origin of Swift J0644.5-5111/FRB 131104 with a different approach.

A long-short burst might be powered by an NS-NS merger event or an BH-NS merger event(Yang et al. 2015). While the former cannot be excluded, we discuss the latter that has also been proposed to be a possible scenario producing FRBs with luminosity $\sim 10^{40}-10^{41}$ $\operatorname{ergs}^{-1}$ (Mingarelli et al. 2015). In the BH-NS merger model, the accretion disk is a cold thin disk (Rosswog 2005) and the duration of the GRB is approximately equal to the viscosity timescale $t_{\mathrm{vis}}$ of the accretion disk

$$
t_{\mathrm{vis}}=\frac{1}{\alpha \Omega_{\mathrm{k}}}\left(\frac{R_{\mathrm{d}}}{H}\right)^{2} \simeq 274 M_{\mathrm{BH}, 1}^{-\frac{1}{2}} R_{\mathrm{d}, 8}^{\frac{3}{2}} \alpha_{-2}^{-1}\left(\frac{h}{0.1}\right)^{-2} \mathrm{~s},
$$

where $\alpha \sim 0.01-0.1$ (Lloyd-Ronning et al. 2016) is the viscosity of the accretion disk, $\Omega_{k}=\sqrt{G M_{\mathrm{BH}} / R_{\mathrm{d}}^{3}}$ is the Keplerian velocity of the accretion disk, $G$ is the gravitational constant, $M_{\mathrm{BH}}=M_{\mathrm{BH}, 1} \times 10 M_{\odot}$ is the mass of the post-merger $\mathrm{BH}, H=h \times R_{\mathrm{d}}$ is the scale-height of the disk, and $R_{\mathrm{d}}=R_{\mathrm{d}, \mathrm{S}} \times R_{\mathrm{S}}$ is the outer radius of the 
disk with $R_{\mathrm{S}}$ being the Schwarzschild radius of the $\mathrm{BH}$. The numerical simulations performed by Rosswog et al. (2004) have shown that the radius of the outer disk can be as large as $600 \mathrm{~km}$.

Letting $h \equiv H / R_{\mathrm{d}}$ and adopting $H=0.1 R_{\mathrm{d}}$, the estimated viscosity timescale $t_{\mathrm{vis}}$ can be approximately equal to the duration of Swift J0644.5-5111. Therefore, we suggest that Swift J0644.5-5111 might be a long-short burst originating from a NS-BH merger event that could also have produced FRB 131104.

While FRB 131104 might be powered by a NS-BH merger event, we note that only a minor fraction of FRBs can be supposed to be associated with an NS-NS/BH merger events since the FRB rate is much higher than the predictions for compact object coalescence rate. In other words, FRB 131104 would have to be fundamentally different than other FRBs.

\subsection{The contribution from the $U V$ emission of a kilonova}

The merger of a NS-NS binary or a BH-NS binary would eject $\sim 0.001-0.1 M_{\odot}$ of neutron-rich material and synthesize some $r$-process heavy elements. The radioactive decay of these $r$-process elements could heat the ejecta and yield UV-optical-IR radiation (named "kilonova" e.g., Li \& Paczyński 1998; Metzger et al. 2010; Barnes \& Kasen 2013).

In the BH-NS merger scenario, the contribution from the emission of a kilonova must be taken into account. We use the equations derived by Kawaguchi et al. (2016) to calculate the multi-band emission from a kilonova. The parameters adopted are listed below: the optical opacity $\kappa=10 \mathrm{~cm}^{2} \mathrm{~g}^{-1}$, the ejecta mass $M_{\mathrm{ej}}=0.02 M_{\odot}$, the average velocity of the mass $v_{\mathrm{ej}}=0.18 c$, the minimum velocity of the mass $v_{\mathrm{ej}, \mathrm{min}}=0.02 c$, the efficiency of thermalization $\epsilon_{\mathrm{th}}=0.5$. The multi-band $(U, R, I$, and $Z$ ) light curves are plotted in Fig. 6. We find that the $U$ band emission $\left(<10^{-2} \mu \mathrm{Jy}\right)$ is significantly lower than the upper limit $(\sim 30 \mu \mathrm{Jy})$ of the $U$-band observation and can be neglected.

\section{CONCLUSIONS}

The long-duration $\left(T_{90} \approx 377 \mathrm{~s}\right)$ gamma-ray transient Swift J0644.5-5111 was supposed to be associated with FRB 131104 (DeLaunay et al. 2016). The followup observations for Swift J0644.5-5111 got the upper limits of radio $(5.5 \mathrm{GHz}$ and $7.5 \mathrm{GHz}), U$-band and $\mathrm{X}$-ray radiation. Previous research used the standard GRB afterglow model to constrain the properties of the ISM surrounding the progenitor system of Swift J0644.5$5111 /$ FRB 131104. These works have neglected the jet effect and the jet evolution effect.

In this paper, we have adopted the unified dynamic model for the multi-band afterglows of GRBs and considered the upper limits of the observations for the electromagnetic counterparts at $5.5 \mathrm{GHz}, 7.5 \mathrm{GHz}, U$-band, and X-ray to constrain two most important parameters, $\varepsilon_{\mathrm{B}}$ and $n$. To explain how our models are fundamentally different than those presented elsewhere, we presented Table 2 that can differentiate our model and the previous models.

Using this model, we found that when $\varepsilon_{\mathrm{B}}=0.0001$,

7 It should be noted that the distances of FRBs are orders of $n$ cannot be constrained (in the jet case) or $n \leq 6.5 \times$ $10^{-2} \mathrm{~cm}^{-3}$ (in the isotropic case); when $\varepsilon_{\mathrm{B}}=0.001$, $n \leq 6.5 \times 10^{-2} \mathrm{~cm}^{-3}$ (jet) or $n \leq 9.0 \times 10^{-2} \mathrm{~cm}^{-3}$ (isotropic); when $\varepsilon_{\mathrm{B}}=0.01, n \leq 7.4 \times 10^{-4} \mathrm{~cm}^{-3}$ (jet) or $n \leq 2.0 \times 10^{-4} \mathrm{~cm}^{-3}$ (isotropic); when $\varepsilon_{\mathrm{B}}=0.1, n \leq$ $6.3 \times 10^{-5} \mathrm{~cm}^{-3}$ (jet) or $n<3.0 \times 10^{-6} \mathrm{~cm}^{-3}$ (isotropic). It can be found that differences between the jet case and isotropic case are so obvious that the jet effect must be taken into account. If we have more accurate afterglow data, the multi-band light curves produced by a jet and an isotropic outflow can be distinguished since the former emission would produce a jet break in light curves.

Furthermore, we plotted the $n-\varepsilon_{\mathrm{B}}$ contour sub-figures (see Fig. 5) for the upper limits at $5.5 \mathrm{GHz}, 7.5 \mathrm{GHz}$, $U$-band, as well as X-ray, and found that the inferred upper limits of the density of the environment of the progenitor are consistent with both that of massive stars $\left(n \sim 1-10 \mathrm{~cm}^{-3}\right)$ and neutron stars $\left(n \ll 1 \mathrm{~cm}^{-3}\right.$ and can be as low as $10^{-8} \mathrm{~cm}^{-3}$, e.g., Zheng et al. 2014). Since the duration $T_{90} \approx 377 \mathrm{~s}$ and the massive-star scenario is excluded, the remaining possibilities are that Swift J0644.5-5111 is an off-axis short GRB with an extended emission (Dai et al. 2016a) or a long-short burst. Besides, the results above suggest that the environmental properties of Swift J0644.5-5111/FRB 131104 favor a BH-NS merger scenario, supporting the conclusion of Murase et al. (2017).

Since the BH-NS merger must eject $0.001-0.1 M_{\odot}$ of neutron-rich material and a kilonova powered by the radioactivity of $r$-process elements synthesized in it must be taken into account, we calculated the multi-band emission from the kilonova. By comparing the $U$-band luminosity of the kilonova and the upper limit of the U-band observation, we found that the former is significantly lower than the latter. Therefore we can conclude that the kilonova emission can be neglected and the constraints obtained in section 3 are valid without further modifications.

We caution that the connection between the Swift J0644.5-5111/FRB 131104 is tenuous and all other FRBs do not have gamma-ray counterparts. Based on the large distance inferred from the DM of FRB 131104 and the fact that Swift J0644.5-5111 is a highly offaxis event, the derived isotropic gamma-ray energy of Swift J0644.5-5111 is very high. It would be expected that upcoming multi-band and multi-messenger observations for FRBs could pose more stringent constraints on all relevant parameters, shedding more light on the nature of FRBs. ${ }^{7}$

We thank an anonymous referee for helpful comments and suggestions that have allowed us to improve our manuscript. We thank Yong-Feng Huang, Jin-Jun Geng, and Long-Biao Li for helpful discussion. This work was supported by the National Basic Research Program ("973" Program) of China (grant no. 2014CB845800) and the National Natural Science Foundation of China (grant no. 11573014). S.Q.W., L.D.L., and H.Y. are supported by the China Scholarship Program to conduct research at UCB, UNLV and KSU, respectively. 


\section{REFERENCES}

Abadie, J., Abbott, B. P., Abbott, R., et al. 2010, Classical and Quantum Gravity, 27, 173001

Burke-Spolaor, S., \& Bannister, K. W. 2014, ApJ, 792, 19

Caleb, M., Flynn, C., Bailes, M., et al. 2017, MNRAS, 468, 3746

Connor, L., Sievers, J., \& Pen, U. L. 2016, MNRAS, 458, L19

Dai, Z. G., \& Lu, T. 1998a, MNRAS, 298, 87

Dai, Z. G., \& Lu, T. 1998b, A\&A, 333, L87

Dai, Z. G., Wang, J. S., \& Wu, X. F. 2016a, arXiv:1611.09517

Dai, Z. G., Wang, J. S., Wu, X. F., \& Huang, Y. F. 2016b, ApJ, 829, 27

Dai, Z. G., Wang, J. S., \& Yu, Y. W. 2017, ApJL, 838, L7

DeLaunay, J. J., Fox, D. B., Murase, K., et al. 2016, ApJL, 832, L1

Falcke, H., \& Rezzolla, L. 2014, A\&A, 562, A137

Gao, H., \& Zhang, B. 2017, ApJL, 835, L21

Geng, J. J., \& Huang, Y. F. 2015, ApJ, 809, 24

Gu, W. M., Dong, Y. Z., Liu, T., Ma, R., \& Wang, J. 2016, ApJL, 823, L28

Hassall, T. E., Keane, E. F., \& Fender, R. P. 2013, MNRAS, 436, 371

Huang, Y. F., Dai, Z. G., \& Lu, T. 1999, MNRAS, 309, 513

Huang, Y. F., Dai, Z. G., \& Lu, T. 2000a, MNRAS, 316, 943

Huang, Y. F., Gou, L. J., Dai, Z. G., \& Lu, T. 2000b, ApJ, 543, 90

Hurley, K., Boggs, S. E., Smith, D. M., et al. 2005, Nature, 434, 1098

Kashiyama, K., Ioka, K., \& Mészáros, P. 2013, ApJL, 776, L39

Katz, J. I. 2014, Phys. Rev. D, 89, 103009

Kawaguchi, K., Kyutoku, K., Shibata, M., \& Tanaka, M. 2016, ApJ, 825, 52

Keane, E. F., Johnston, S., Bhandari, S., et al. 2016, Nature, 530, 453

Kulkarni, S. R., Ofek, E. O., Neill, J. D., Zheng, Z., \& Juric, M. 2014, ApJ, 797, 70

Li, L. X., \& Paczyński, B. 1998, ApJL, 507, L59

Li, L.-B., Huang, Y.-F., Zhang, Z.-B., Li, D., \& Li, B. 2017, Research in Astronomy and Astrophysics, 17, 6

Lingam, M., \& Loeb, A. 2017, ApJL, 837, L23

Lloyd-Ronning, N. M., Dolence, J. C., \& Fryer, C. L. 2016, MNRAS, 461, 1045
Lorimer, D. R., Bailes, M., McLaughlin, M. A., Narkevic, D. J., \& Crawford, F. 2007, Science, 318, 777

MacFadyen, A. I., \& Woosley, S. E. 1999, ApJ, 524, 262

Mészáros, P., \& Rees, M. J. 1997, ApJ, 476, 232

Metzger, B. D., Martinez-Pinedo, G., Darbha, S., et al. MNRAS, 2010, 406, 2650

Barnes, J., \& Kasen, D. ApJ, 2013, 775, 18

Mingarelli, C. M. F., Levin, J., \& Lazio, T. J. W. 2015, ApJL, 814, L20

Mottez, F., \& Zarka, P. 2014, A\&A, 569, A86

Murase, K., Mészáros, P., \& Fox, D. B. 2017, ApJL, 836, L6

Palaniswamy, D., Li, Y., \& Zhang, B. 2018, ApJ, 854, L12

Piro, A. L., \& Burke-Spolaor, S. 2017, ApJL, 841, L30

Ravi, V., Shannon, R. M., Bailes, M., et al. 2016, Science, 354, 1249

Rosswog, S. 2005, ApJ, 634, 1202

Rosswog, S., Speith, R., \& Wynn, G. A. 2004, MNRAS, 351, 1121

Santana, R., Barniol Duran, R., \& Kumar, P. 2014, ApJ, 785, 29

Sari, R., Piran, T., \& Narayan, R. 1998, ApJL, 497, L17

Shannon, R. M., \& Ravi, V. 2017, ApJL, 837, L22

Thompson, C., \& Duncan, R. C. 1995, MNRAS, 275, 255

Thornton, D., Stappers, B., Bailes, M., et al. 2013, Science, 341, 53

Totani, T. 2013, PASJ, 65, L12

Usov, V. V. 1992, Nature, 357, 472

Wang, J. S., Yang, Y. P., Wu, X. F., Dai, Z. G., \& Wang, F. Y. 2016, ApJL, 822, L7

Williams, P. K. G., \& Berger, E. 2016, ApJL, 821, L22

Woosley, S. E. 1993, ApJ, 405, 273

Yang, B., Jin, Z. P., Li, X., et al. 2015, Nature Communications, 6,7323

Yang, Y.-P., \& Zhang, B. 2017, arXiv:1712.02702

Zadorozhna, L. V. 2015, Advances in Astronomy and Space Physics, 5, 43

Zhang, B. 2016, ApJL, 827, L31

Zheng, Z., Ofek, E. O., Kulkarni, S. R., Neill, J. D., \& Juric, M. 2014, ApJ, 797, 71 magnitude larger than the distance limits of detections of current and near-Future GW detectors so that the rate of GW events asso- ciated with NS-NS/BH mergers in general is much lower than the FRB rate. 

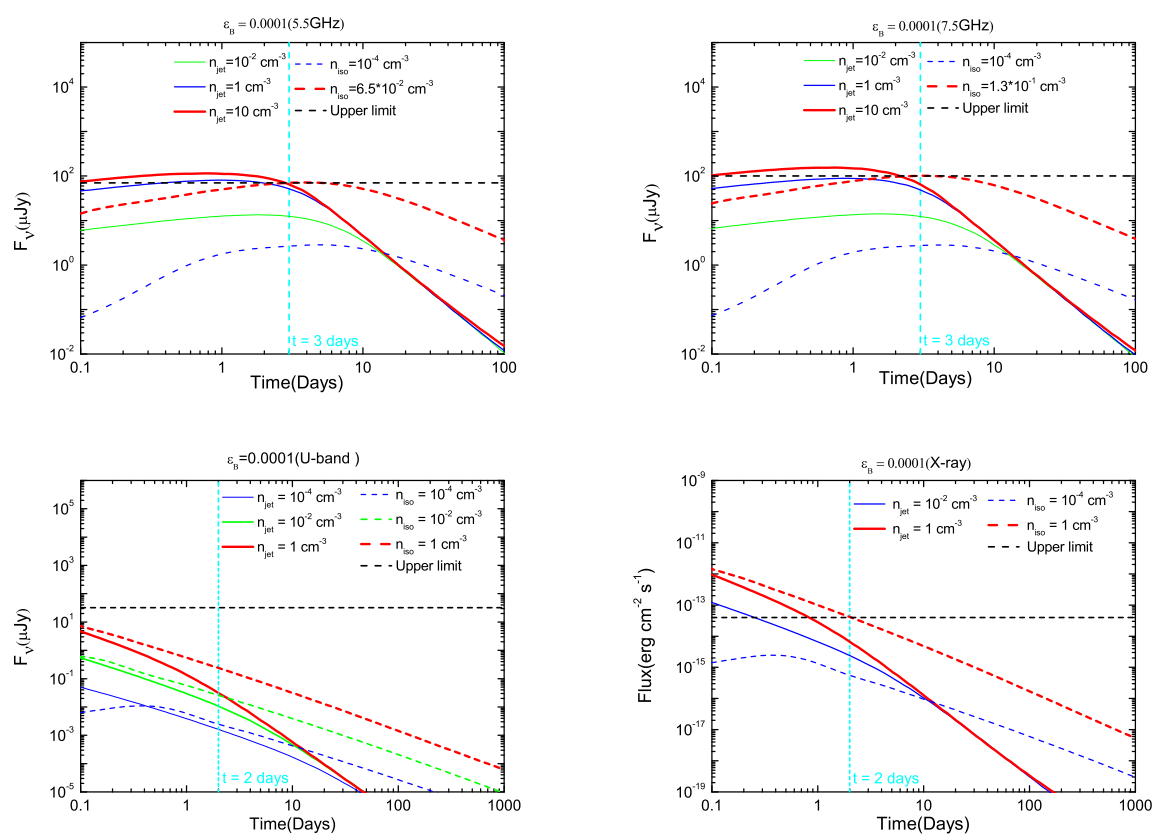

FIG. 1.- The light curves of $5.5 \mathrm{GHz}, 7.5 \mathrm{GHz}$, U-band, and X-ray when the fraction of the shock energy going into the electrons $\epsilon_{B}$ is set to be 0.0001 . The solid lines correspond to the jet case while the dashed lines correspond to the isotropic-explosion case. The black dashed lines represent the detection limits of each band, while the vertical cyan lines represent the start times of observations. The light curves yielded by different densities (indicated in the panels) are represented by different colors, thicknesses and styles.
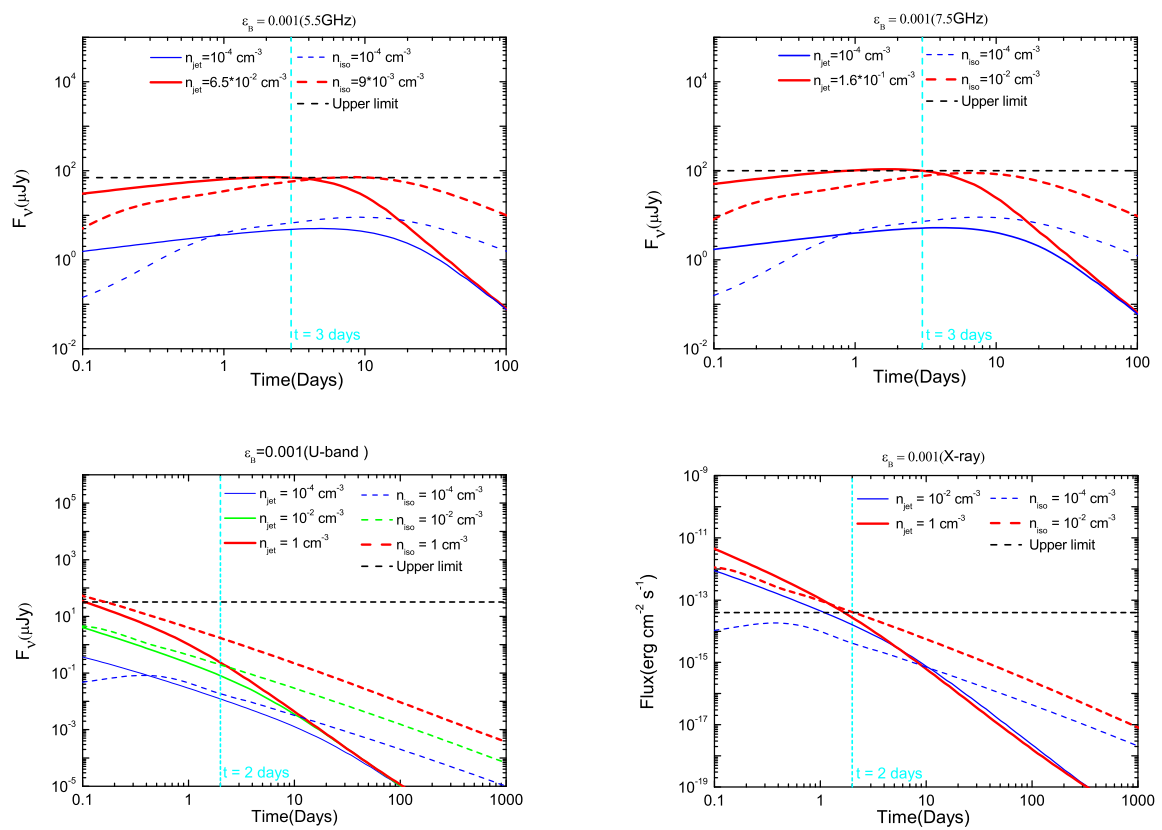

FIG. 2.- Same as in Fig. 1 but $\epsilon_{B}$ is 0.001 . 

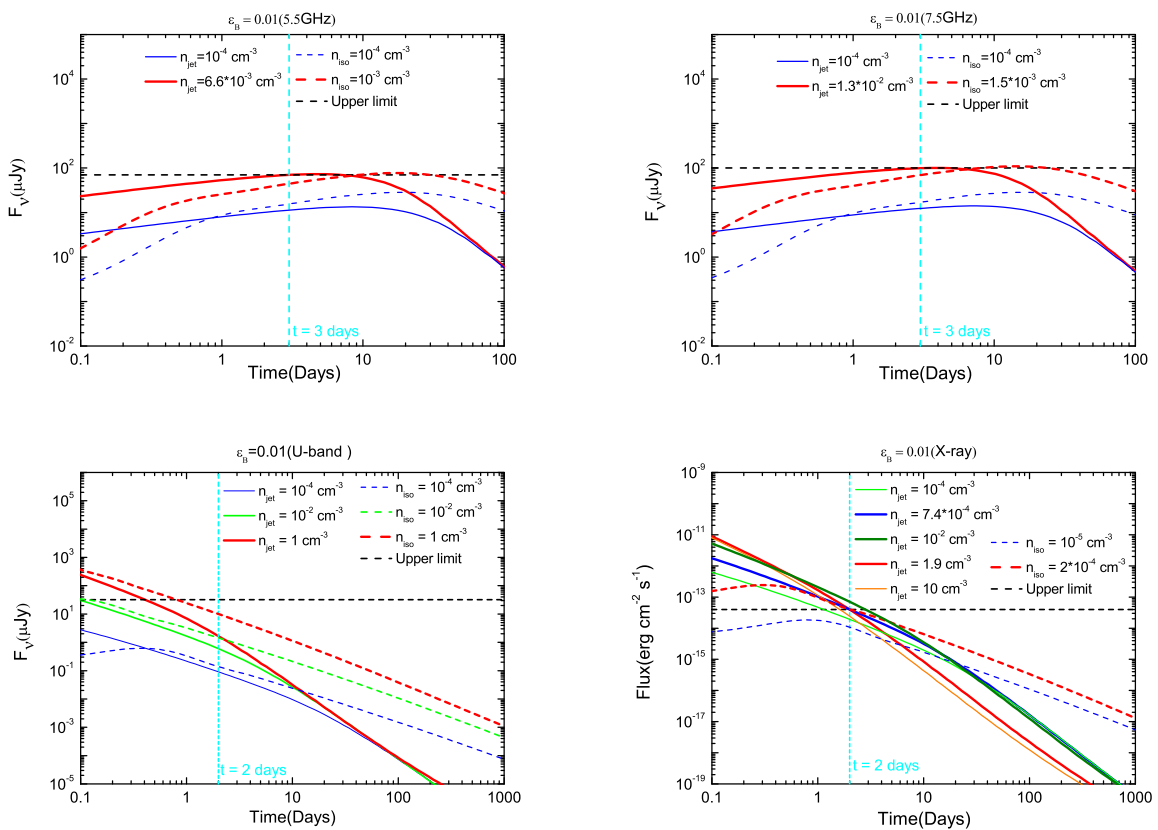

FIG. 3. - Same as in Fig. 1 but $\epsilon_{B}$ is 0.01 . The lines in the last panel are more than those of the remaining panels of Fig. 1 and Fig. 2 since the lines reach the upper limit at $t=2$ days if $n=1.9 \mathrm{~cm}^{-3}$ or $7.4 \times 10^{-4} \mathrm{~cm}^{-3}$ and additional light curves corresponding to other three possible cases $\left(n>1.9 \mathrm{~cm}^{-3}, 7.4 \times 10^{-4} \mathrm{~cm}^{-3}<n<1.9 \mathrm{~cm}^{-3}\right.$, and $\left.n<7.4 \times 10^{-4} \mathrm{~cm}^{-3}\right)$ are given.
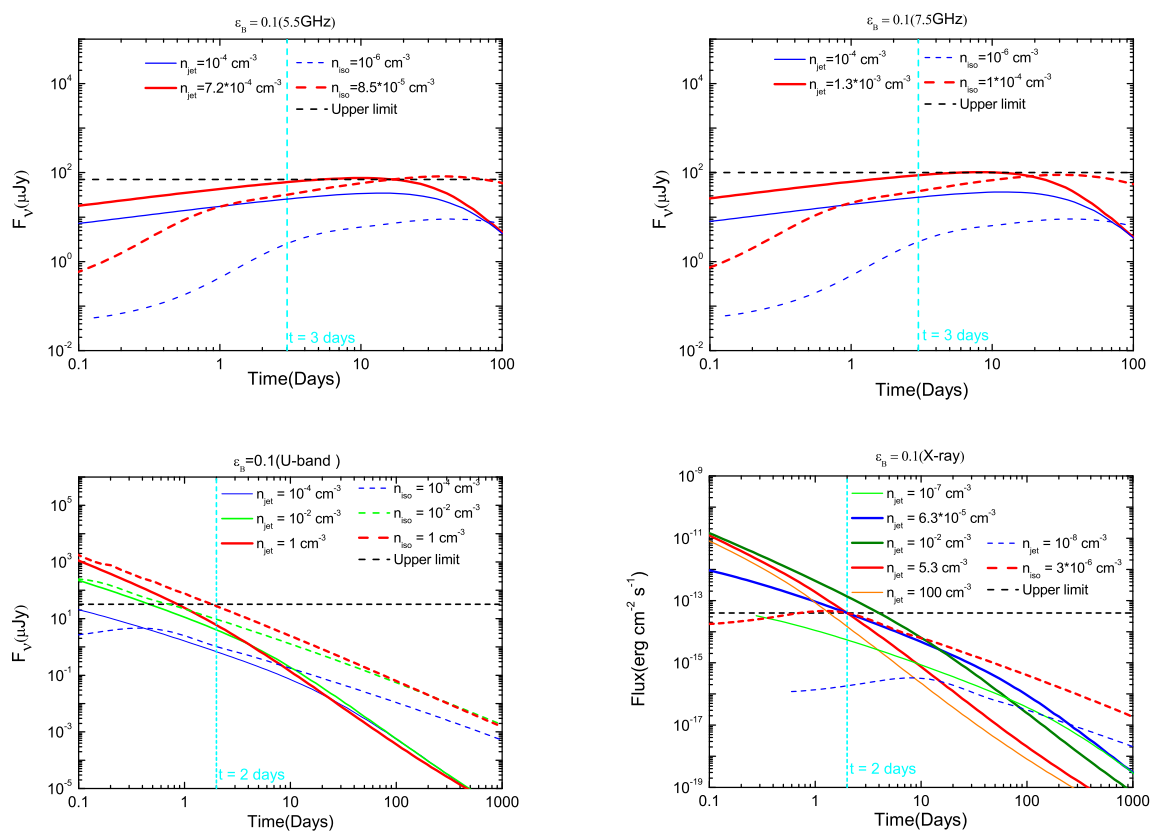

FIG. 4.- Same as in Fig. 3 but $\epsilon_{B}$ is 0.1 . 

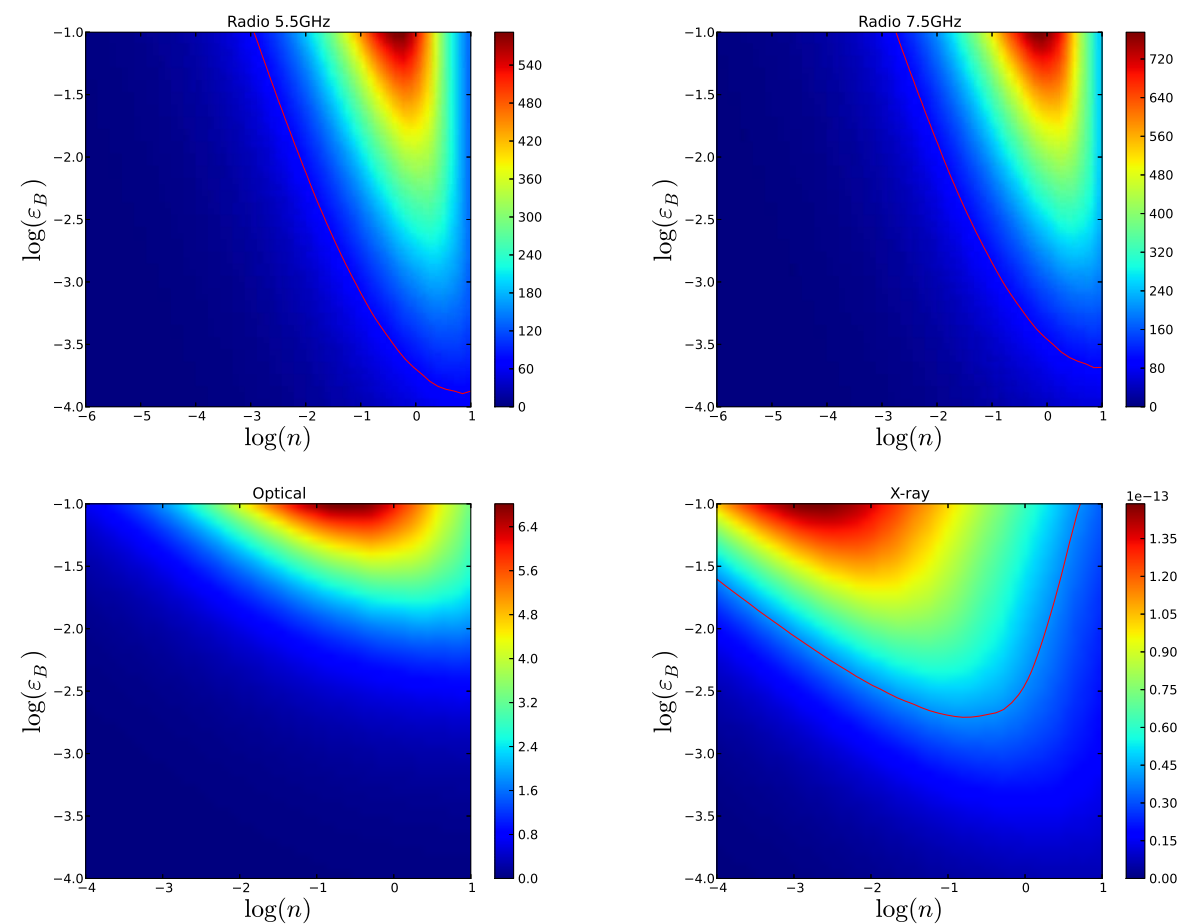

Fig. 5.- Contours for the detected flux at 5.5 GHz, 7.5 GHz, $U$-band, and X-ray. The red lines represent the detect limits. Panel c has no line, indicating that the theoretical $U$-band flux reproduced by all possible parameters are smaller than the detected limit.

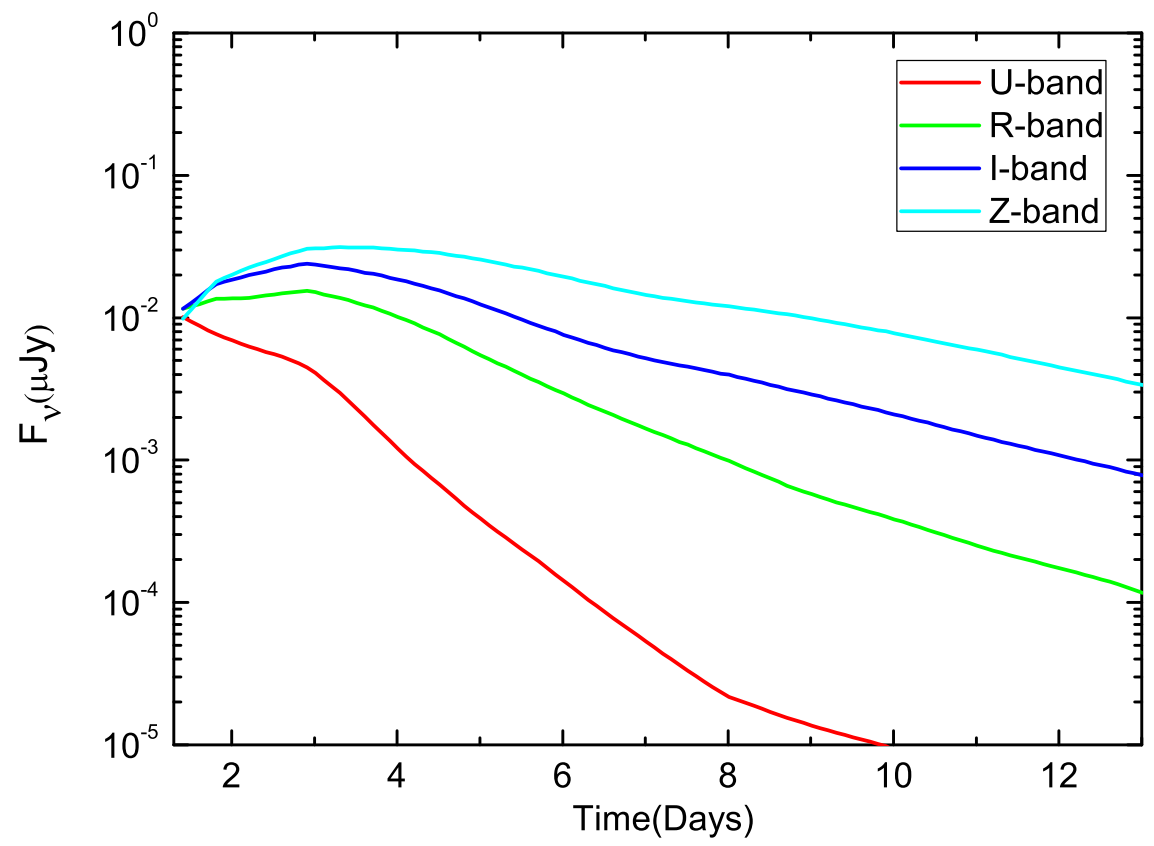

FIG. 6. - The light curves of a kilonova at a redshift of 0.55 . 